\title{
Multi-objective Optimization Algorithm for Multimedia English Teaching (MOAMET) Based on Computer Network Traffic Prediction Model
}

\author{
https://doi.org/10.3991/ijet.v13i03.8372 \\ Weiwei Feng \\ Xi'an University, Xi'an, Shaanxi, China \\ kimbly1212@qq.com
}

\begin{abstract}
In order to solve the problem of multi-objective optimization for multimedia English teaching, this paper proposes a multi-objective optimization algorithm for multimedia English teaching (MOAMET) based on computer network traffic prediction model, which is based on the computer network traffic prediction model strategy. This algorithm establishes time series for individuals correlated to same reference points, and for such time series through computer network traffic model optimizes multimedia English teaching objectives. Meanwhile, it feeds back the prediction error of the historical moment to the current prediction to improve the accuracy of the optimization, and adds disturbance in each optimized individual to increase the diversity of initial multimedia English teaching so as to speed up the convergence speed of the algorithm in the new environment. Through experiments it teats the algorithm, also makes comparison and analysis with two existing algorithms, the results show that the proposed algorithm can maintain good performance in dealing with multi-objective optimization for multimedia English teaching.
\end{abstract}

Keywords-computer network; traffic prediction model; multimedia English teaching; multi-objective optimization

\section{Introduction}

There are a large number of complex multi-objective optimization problems with time-varying in the field of multimedia English teaching[1], that is multi-objective function and constraint conditions of multimedia English teaching are not only related to decision-making variables but also change with time, so the optimal solution or optimal frontier also changes over time[2-3]. Many scientific research problems, such as machine learning, double-layer optimization, constraint optimization etc, can also be treated as the problems of multi-objective optimization for multimedia English teaching for treatment[4-5]. However, this type of problem has multiple timedependent and conflicting goals, and the optimal frontier changes with time[6]. Therefore, it is difficult to design effective solutions. The appearance of Evolutionary algorithms (EAs) provides a new way for solving the problem of dynamic optimization[7]. Compared with the traditional optimization algorithms, EAs has features such as high 
parallel, self-organizing, self-adaptive, self-learning etc, and is a global optimization algorithm with wide applicability[8 -9]. Therefore, the use of EAs to solve dynamic optimization problems has attracted more and more attention[10].

To sum up the above problems, this paper proposes a multi-objective optimization algorithm for multimedia English teaching (MOAMET) based on structural computer network traffic prediction model. This algorithm respectively establishes time series for the individuals correlated with the same reference point, makes a prediction on the traffic under computer network environment, thus can improve the optimization precision when optimizing the multi-objective optimization for multimedia English teaching. Compared with the existing algorithms, the proposed algorithm can quickly optimize the problem of multi-objective optimization for multimedia English teaching and have good environmental adaptability.

\section{Description of problem of multi-objective optimization for multimedia English teaching}

In general, the problem of multi-objective optimization for multimedia English teaching can be defined as follows:

$$
\left\{\begin{aligned}
\min f(x, t) & =\left\{f_{1}(x, t), f_{2}(x, t), \mathrm{L}, f_{M}(x, t)\right\} \\
\text { s.t.g } g(x, t) & \leq 0 \\
h(x, t) & =0 \\
x & \in D
\end{aligned}\right.
$$

Where, $t$ is the time variable, $x=\left(x_{1}, x_{2}, \mathrm{~L}, x_{n}\right)^{T}$ is the $n$-dimensional decisionmaking variable, $g$ and $h$ are respectively the inequality and the equality constraint, $D$ is the search space, $f$ is a $M$-dimensional objective function dependent on time $t$ variation.

Definition 1. For a certain time (environment) $t$, we call vector $u_{t}=\left(u_{1}^{t}, u_{2}^{t}, \mathrm{~L}, u_{M}^{t}\right)^{T}$ multi-objective dominating vector $v_{t}=\left(v_{1}^{t}, v_{2}^{t}, \mathrm{~L}, v_{M}^{t}\right)^{T}$, if and only if $\forall i \in\{1,2, \mathrm{~L}, M\}, u_{i}^{t} \leq v_{i}^{t}$ and $\exists i \in\{1,2, \mathrm{~L}, M\}, u_{i}^{t} \leq v_{i}^{t}$, make $u_{i}^{t}<v_{i}^{t}$, where $M$ is the number of the objective function.

Definition 2. For a certain time (environment) $t$, we call the vector $x_{u} \in D$ is the multi-objective optimal solution to the problem (1), if and only if there is no $x_{v} \in D$, make $v_{t}=f\left(x_{v}, t\right)$ multi-objective dominating $u_{t}=f\left(x_{u}, t\right)$.

The set composed of all multi-objective optimal solutions to the optimization problem is called the multi-objective optimal solution set; accordingly, the corresponding set of objective function values forms the multi-objective optimal frontier. According to the multi-objective optimal solution set and its frontier changes over time, dynamic multi-objective problems can be divided into the following four categories: 
1. Multi-objective optimal solution set changes with time while the multi-objective optimal frontier does not change with time;

2. Multi-objective optimal solution set and multi-objective optimal frontier change with time;

3. Multi-objective optimal solution set does not change with time while the multiobjective optimal frontier changes with time;

4. Multi-objective optimal solution set and multi-objective optimal frontier do not change with time.

\section{Multi-objective optimization algorithm for multimedia English teaching}

\subsection{Multimedia English teaching history information reuse}

When the environment changes, use multimedia English teaching history information reuse to predict the new computer network traffic, the premise is to reasonably select effective multimedia English teaching history information reuse. In the dynamic single-objective optimization problem, it is only necessary to predict the current sole the optimal solution or partial local optimal solution, the selection of reuse of multimedia English teaching history information is relatively simple and effective. While the problem of multi-objective optimization for multimedia English teaching needs to optimize the multi-objective optimal solution set, the reuse of multimedia English teaching history information reuse is more difficult.

This paper proposes a fusion of structured reference points and multimedia English teaching individual correlation strategies to record the English teaching history information, and use the information reuse to form multimedia English teaching methods in the initial new environment. The individuals under different environments and correlated under the same reference point are taken as a time series, for each time series use computer network prediction model to predict individuals in the new environment. In this paper, the adopted designed reference points are those uniformly distributed in the hyperplane, the set of reference points is recorded as $Z$, as shown in formula (2).

$$
Z:\left\{\begin{array}{l}
Z_{i}=\left(z_{i}^{1}, z_{i}^{2}, \mathrm{~L}, z_{i}^{M}\right) \\
z_{i}^{j} \in\left\{\frac{0}{p}, \frac{1}{p}, \mathrm{~L}, \frac{p}{p}\right\}, \sum_{j=1}^{M} z_{i}^{j}=1
\end{array}\right.
$$

Where, $i=1,2$, $\mathrm{L}, H, H=\left(\begin{array}{c}M+p-1 \\ p\end{array}\right)$ is the number of reference points, $M$ is the number of objective functions, $p$ is the number of coordinate segments per dimension. In general, set $H \approx N, N$ the size for multimedia English teaching.

For the two-objective dynamic optimization problem solved in this paper, the target size of multimedia English teaching is set to 100, according to the setting method 
of above reference point, the number of reference points generated $H$ is about 100 . The structure distribution of reference point is shown in Figure 1.

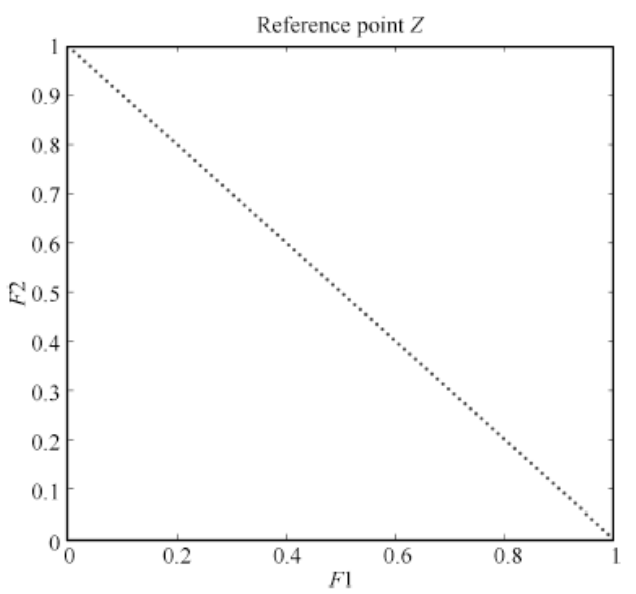

Fig. 1. Reference Points of Multi-Objective Optimization for Multimedia English Teaching

\subsection{Strategy for multi-objective optimization for multimedia English teaching}

Each reference point forms time series at different moments according to the multiobjective optimal solution $\left\{\mathrm{L}, x_{t-3}^{i}, x_{t-2}^{i}, x_{t-1}^{i}, x_{t}^{i}\right\}(i=1,2, \mathrm{~L}, N)$ of Algorithm 1. The series reflects the variation discipline of the optimal solution, while in the same environment, the individuals correlated with different reference points reflect the distribution of multi-objective optimal solution sets in the current environment. Therefore, the time series of each reference point describes the movement of the multi-objective solution set of the problem, and the prediction of the location of the multi-objective solution set at moment $t+1$ can be expressed as

$$
x_{t+1}^{i}=f\left(x_{t}^{i}, x_{t-1}^{i}, \mathrm{~L}, x_{t-K+1}^{i}, t\right)
$$

Where, $f$ represents the network traffic prediction model, $i=1,2, \mathrm{~L}, H$ represents the reference point. In order to reduce the complexity of the optimization, we select computer network traffic model for prediction. Establish the prediction model by taking the information of the first two moments as a multimedia English teaching historical information reuse. From the third moment, for the individuals correlated to the same reference point adopt the following model to predict new individuals.

$$
x_{t+1}^{i}=x_{t}^{i}+\left(x_{t}^{i}-x_{t-1}^{i}\right)+\varepsilon
$$


Where, $\varepsilon=x_{t}^{i}-x_{t}^{i}$ is the prediction error of the moment $t$. Feedback the prediction error of the moment $t$ to the prediction of the moment $t+1$ to improve the accuracy of the optimization.

Table 1. Individual Correlation Algorithm

\begin{tabular}{ll}
\hline Algorithm 1 & \multicolumn{1}{c}{ Individual correlation algorithm } \\
\hline Step 1 & $\begin{array}{l}\text { for } i=1: H \mathrm{do} / / H \text { The number of reference points } \\
\text { Link the reference point and original point as reference line for this } \\
\text { reference point } \\
\text { end } 2\end{array}$ \\
Step 3 & $\begin{array}{l}\text { for } i=1: N \text { do// } N \text { The number of individuals for multimedia English } \\
\text { teaching } \\
\text { ftep } 4\end{array}$ \\
for $j=1: H$ do 5 & Calculate the distance between each individual and the reference line \\
Step 6 & end \\
Step 7 & $\begin{array}{l}\text { The reference point with the lowest vertical distance from the individual } \\
\text { is recorded as the correlated reference point } \\
\text { Step } 8\end{array}$ \\
Step 9 &
\end{tabular}

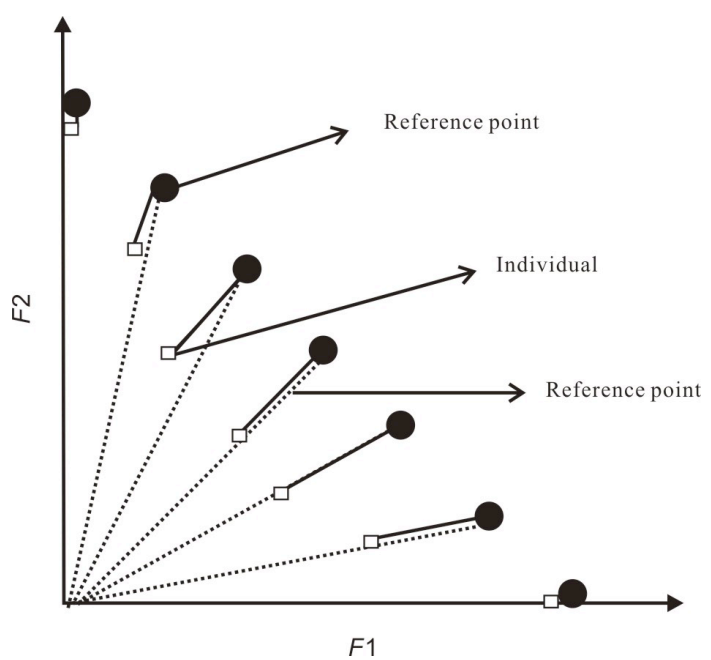

Fig. 2. Multi-objective Optimization Problem Individual And Reference Point Correlation

In addition, in order to maintain the diversity of multimedia English teaching, this paper presents Gaussian disturbance $\zeta$ around each predicted individual to increase the diversity of multimedia English teaching predictions, that is:

$$
x_{t+1}^{i}=x_{t+1}^{i}+\varsigma
$$

The gaussian disturbance is defined as follows: 


$$
\varsigma: N\left(0, \delta^{2}\right)
$$

Where, $\delta$ is the standard deviation:

$$
\delta^{2}=\frac{1}{4 n}\left\|x_{t}-x_{t-1}\right\|_{2}^{2}
$$

Where, $n$ is the dimension of decision-making variables.

\subsection{MOAMET algorithm steps}

MOAMET algorithm adopts static multi-objective optimization algorithm NSGAII algorithm as the basic framework, MOAMET algorithm pseudo-code is shown in Table 2.

\begin{tabular}{|c|c|}
\hline Algorithm 2 & MOAMET algorithm pseudo-code \\
\hline Step 1 & $\begin{array}{l}\text { Parameters and multimedia English teaching initialization: set initialization } \\
\text { parameters, the time constant } \tau_{t} \text {, multimedia English teaching size pop, evolu- } \\
\text { tion algebra max gen, and in the decision-making space randomly generate scale } \\
\text { pop initial multimedia English teaching } p_{0}^{t} \text {. } \\
\text { Let } t=0, T=0 \text {, gen }=0\end{array}$ \\
\hline Step 2 & $\begin{array}{l}\text { Environmental detection: according to formula (7) calculate } \eta(t) \text {, if } \eta(t)\langle\eta \\
\text { then go to Step 3, otherwise go to Step } 4 \text {. }\end{array}$ \\
\hline Step 3 & $\begin{array}{l}\text { The environment has not changed, evolutionary operations update the parent } \\
\text { individual. }\end{array}$ \\
\hline Step 3.1 & $\begin{array}{l}\text { Evolutionary operation: with a certain crossover probability } p_{c} \text {, mutation proba- } \\
\text { bility } p_{m} \text {, make an evolutionary operation for the current parent individual } \\
p_{t}^{\text {gen }} \text {, produce new multimedia English teaching } \Omega_{t}^{g e n} .\end{array}$ \\
\hline Step 3.2 & $\begin{array}{l}\text { Sort quickly for } \Omega_{t}^{\text {gen }} \mathrm{U} p_{t}^{\text {gen }} \text {, and according to reference point correlation } \\
\text { select the individual } p_{t+1}^{g e n} \text { as the next generation of individuals, go to Step } 5 .\end{array}$ \\
\hline Step 4 & $\begin{array}{l}\text { The environment changes, resulting in predictive multimedia English teaching } \\
\text { response changes }\end{array}$ \\
\hline Step 4.1 & $\begin{array}{l}\text { Produce predictive multimedia English teaching, fusion prediction model shown } \\
\text { in formula (4), produce predictive multimedia English teaching with multimedia } \\
\text { English teaching size as pop, and take it as initial multimedia English teaching for } \\
\text { the algorithm of the next moment. }\end{array}$ \\
\hline Step 4.2 & Store multimedia English teaching history information reuse, go to Step 5 . \\
\hline Step 5 & $\begin{array}{l}\text { Determine whether to meet the algorithm stop condition, if met stop; otherwise, } \\
t=t+1 \text {, go to Step } 2 \text {. }\end{array}$ \\
\hline
\end{tabular}

Table 2. MOAMET Algorithm Pseudocode 


\section{Experimental simulation and result analysis}

In this paper, we select four standard test questions for multi-objective optimization for multimedia English teaching as FDA1, FDA3, FDA4, FDA5 to do simulation experiments, and make comparison research with DNSGA-II algorithm and DSS algorithm.

\subsection{Test function}

In the four types of dynamic multi-objective, when the environment changes, the first and second types of problems are more difficult to optimize the optimal solution in the new environment. The algorithm proposed in this paper mainly solves such problems. In the standard test function adopted in this paper, FDA1 belongs to the first type in the dynamic problem classification. When the environment changes, the multi-objective optimal frontier surface remains unchanged, while the multi-objective optimal solution changes with the environment change. FDA2 belongs to the second type of problems in the dynamic problem classification, when the environment changes, both the multi-objective optimal frontier surface and the multi-objective optimal solution change with the environment change. FDA1 and FDA3 multiobjective frontier surface is a concave curved surface. FDA4 and FDA5 multiobjective frontier surface is a convex curved surface, and the multi-objective optimal solution changes with the environment change. However, FDA5's multi-objective optimal frontier surface is a second type of problem with the change of the environment, while FDA4 is the first type of problem.

\subsection{Parameter settings}

1. Problem parameter setting: the test function environment change range $\tau_{T}=5$, the decision-making variable dimension $n=20$. The environment change frequency of each problem is to change an environment for 30 generations. When the algorithm runs to 300 generations stop, there is a total of 10 environments, each algorithm runs independently 30 times.

2. The population size is set to 100 .

3. The proposed algorithm and DNSGA-II algorithm are designed with NSGA-II as the framework, with a crossover probability of 0.9 and a mutation probability of 0.1 .

4. DSS algorithm is made with DE algorithm as the framework.

\subsection{Evaluation indicators}

The goal of multi-objective optimization algorithm for multimedia English teaching is to converge as much as possible to dynamic changing multi-objective frontier $P^{*}(t)$ in dynamic environment, and to maintain the diversity of solution set $S(t)$. 
This paper adopts the indicators of inverse generation distance (IGD) and hypervolume ratio (HVR) to evaluate the overall performance of the proposed algorithm, where IGD is defined as follows:

$$
\begin{gathered}
I G D(t)=\frac{\sum_{i=1}^{\left|P^{*}(t)\right|} d_{i}}{\left|P^{*}(t)\right|} \\
d_{i}=\min _{k=1}^{|S(t)|} \sqrt{\sum_{j=1}^{M}\left(f_{j}^{*(i)}-f_{j}^{(k)}\right)^{2}}
\end{gathered}
$$

$I G D(t)$ reflects the convergence of the algorithm and the diversity of multimedia English teaching. The ideal $I G D(t)$ value is zero, indicating that $S(t)$ has obtained the best convergence and diversity. The hypervolume ratio (HVR) is an improvement derived from the hypervolume (HV), reflecting the ability of the algorithm to maintain diversity, calculated as shown in formula (9):

$$
H V R(t)=\frac{H V(S(t))}{H V\left(P^{*}(t)\right)}
$$

Where

$$
H V=\operatorname{volume}\left(\mathrm{U}_{i=1}^{|S|} v_{i}\right)
$$

$H V R(t)$ can give the information for the algorithm maintaining the diversity, when $S(t)$ and $P^{*}(t)$ coincide, the maximum value of $H V R(t)$ is 1 . Therefore, $\operatorname{HVR}(t)$ is larger, indicating that the diversity of the algorithm is better.

\subsection{Simulation results analysis}

The mean values of the HVR performance indicators of the four standard test functions mentioned in the paper using 3 algorithms independently run for 30 times are shown in Fig.7 10. Table 4 shows the mean and variance of HVR performance indicators in 10 environments, which can be used to compare the stability of the algorithm in various environments.

The Fig.7 10 show HVR performance indicators of the test functions FDA1 FDA5, respectively. It can be intuitively seen from the figure that the HVR of the MOAMET algorithm is significantly higher than that of the DSS algorithm and the DNSGA-II algorithm, indicating that the MOAMET algorithm can better maintain the diversity of multimedia English teaching; at the same time, it can be seen from Table 4 that the HVR performance indicators of the MOAMET algorithm in this paper 
changes little in each environment, indicating that the proposed algorithm can better adapt to the dynamic changes in different environments.

Through the statistical analysis results of IGD and HVR performance evaluation indicators of the algorithm, the MOAMET algorithm proposed in this paper obtains better convergence speed and convergence degree, the multi-objective optimal solution obtained is evenly distributed in the target space, and has good ability to maintain the diversity of multimedia English teaching.

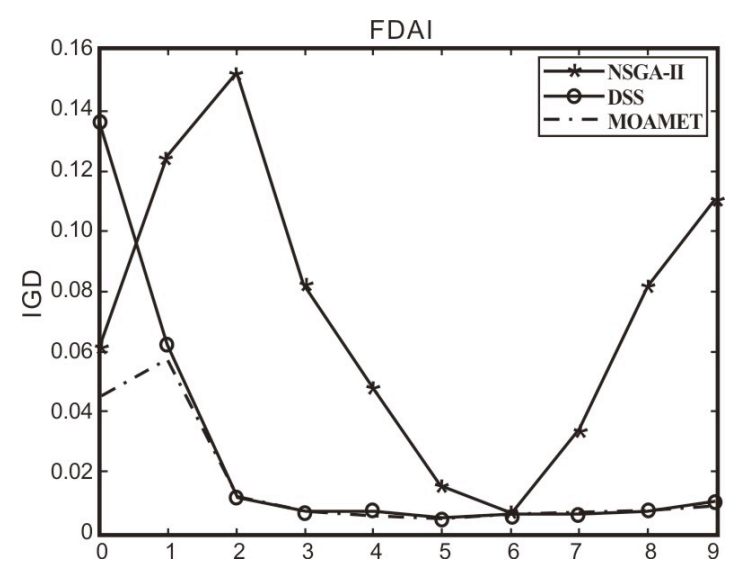

Fig. 3. IGD Mean of FDA1

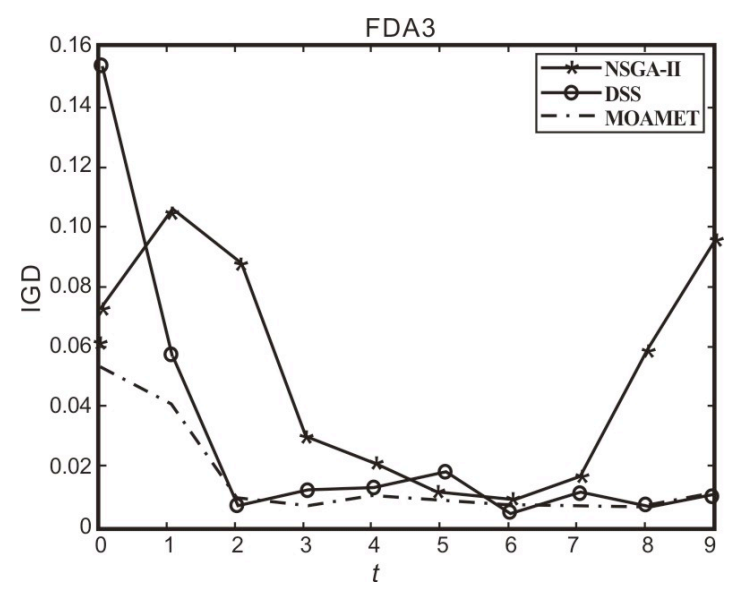

Fig. 4. IGD Mean of FDA3 


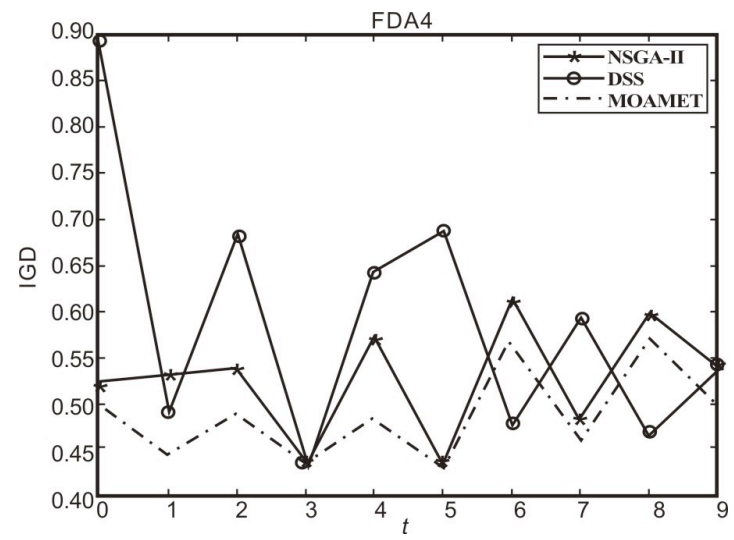

Fig. 5. IGD Mean of FDA4

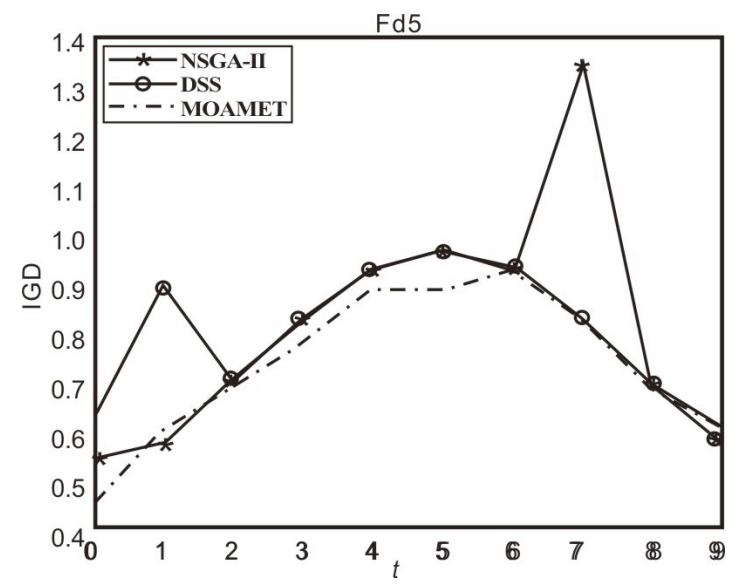

Fig. 6. IGD Mean of FDA5

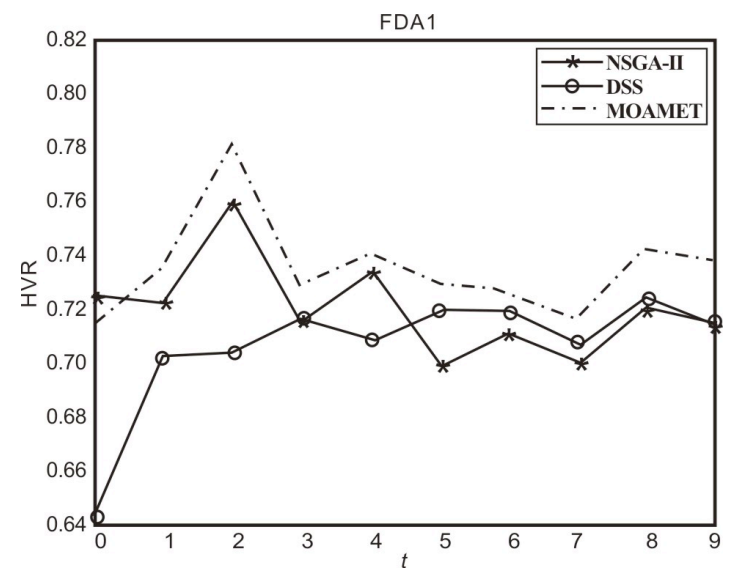

Fig. 7. HVR Mean of FDA1 
Paper-Multi-objective Optimization Algorithm for Multimedia English Teaching (MOAMET) Based ...

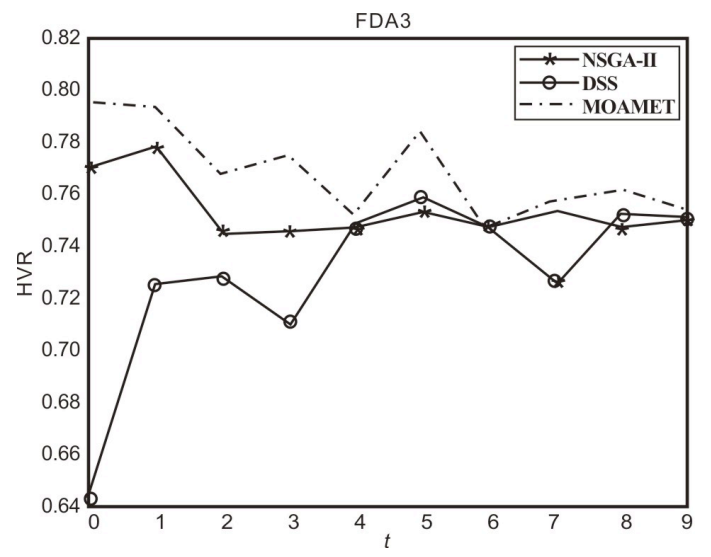

Fig. 8. HVR Mean of FDA3

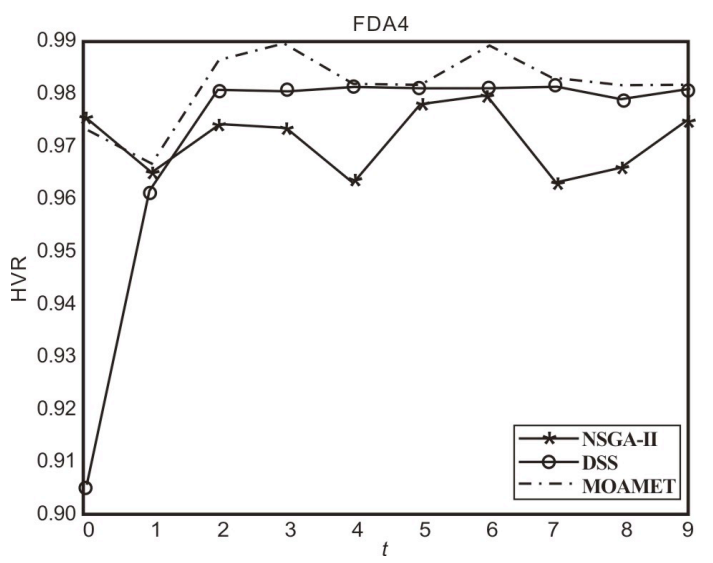

Fig. 9. HVR Mean of FDA4

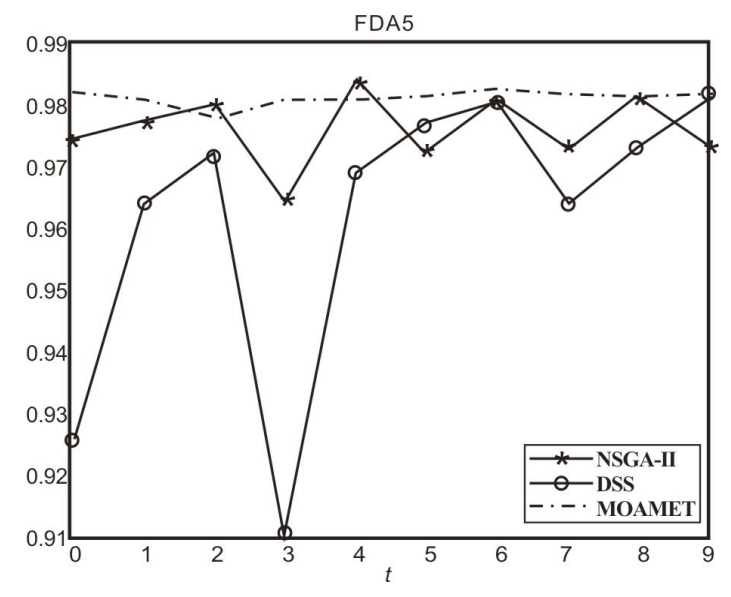

Fig. 10.HVR Mean of FDA5 
3) Experimental Analysis: the analysis of IGD and HVR performance evaluation indicators show that the proposed algorithm is superior to DSS algorithm and DNSGA-II algorithm. From the initial (predictive) multimedia English teaching after the change of environment analyze the superiority of the proposed algorithm. Figure 11-14 gives the predictive multimedia English teaching at the moment of $t=1$ to $t=5$. It is obvious from the figure that when the environment changes, MOAMET's solution for predictive multimedia English teaching is closer to the true solution compared with the DSS algorithm and DNSGA-II algorithm, so that it can quickly converge to the optimal solution in the evolutionary process.

\section{Conclusions}

This paper presents a multi-objective optimization algorithm for multimedia English teaching (MOAMET) based on computer network traffic prediction model. This algorithm uses multimedia English teaching network traffic model to predict the potential distribution area of the optimal solution in a new environment, and improves the ability of the algorithm adapting to environmental changes. For the extraction of multimedia English teaching history information reuse, this paper adopts the individuals correlated to the structural reference points to establish time series, uses the computer network traffic model and prediction error feedback to optimize the multiobjective for multimedia English teaching, and increase the Gaussian disturbance to maintain the diversity for the prediction of multimedia English teaching. The experimental results show that the proposed method can adapt to the change of environment in time and has good portability.

\section{$6 \quad$ References}

[1] Papineni K, Roukos S, Ward T, Zhu Wei-Jing . BLEU : A method for automatic evaluation of machine translation// Proceedings of the 40th Annual Meeting of the Association for Computational Linguistics(ACL). Philadelphia , USA, $2002: 311-318$

[2] Lutz T . Stochastische texte . Augenblick, 1959, 4(1):3-9

[3] Manurung H.Chart generator for rhythm patterned text// Proceedings of the 1st International Workshop on Literature in Cognition and Computer.Tokyo,Japan,1999:15-19

[4] Gervás P. WASP:Evaluation of different strategies for the automatic generation of spanish verse// Proceedings of the AISB00Symposium on Creative \& Cultural Aspects and Applications of AI \&Cognitive Science.Birmingham,UK,2000:93-100

[5] Oliveira H G,Cardoso F A,Pereira F C.Tra-la-Lyrics: An approach to generate text based on rhythm//Proceedings of the 4th International Joint Workshop on Computational Creativity. London, UK,2007:47-55

[6] Kempe V,Levy R,Graci C.Neural networks as fitness evaluators in genetic algorithms:Simulating human creativity// Proceedings of the 23rd Annual Conference of the Cognitive Science Society. Edinburgh,Scotland,2001:1221

[7] Manurung H.An Evolutionary Algorithm Approach to Poetry Generation [Ph. D. dissertation]. University of Edinburgh, Edinburgh,UK,2003 
Paper-Multi-objective Optimization Algorithm for Multimedia English Teaching (MOAMET) Based ...

[8] Gervás P.An expert system for the composition of formal Spanish poetry. Journal of Knowledge-Based Systems, 2001,14(3-4):181-188 https://doi.org/10.1016/S0950$\underline{7051(01) 00095-8}$

[9] Díaz-Agudo B,Gervás P,González-Calero P A.Poetry generation in colibri//Proceedings of the 6th European Conference on Advances in Case-Based Reasoning. London, UK,2002:73-102

[10] Zhou Chang-Le, You Wei, Ding Xiao-Jun. Genetic algorithm and its implementation of automatic generation of Chinese SONGCI. Journal of Software,2010,21(3):427-437(in Chinese) https://doi.org/10.3724/SP.J.1001.2010.03596

[11] Jiang Long, Zhou Ming . Generating Chinese couplets using a statistical MT approach// Proceedings of the 22nd International Conference on Computational Linguistics. Manchester, England, 2008: 377-384

[12] He Jing, Zhou Ming, Jiang Long . Generating Chinese classical poems with statistical machine translation models// Proceedings of the 26th AAAI Conference on Artificial Intelligence . Toronto, Canada, 2012:1650-1656

[13] Genzel D , Uszkoreit J , Och F. "Poetic" statistical machine translation: Rhyme and meter// Proceedings of the 2010 Conference on Empirical Methods in Natural Language Processing. Massachusetts, USA, 2010:158-166

[14] Och F J. Minimum error rate training in statistical machine translation//Proceedings of the 41st Annual Meeting of the Association for Computational Linguistics(ACL 2003). Sapporo, Japan, 2003:160-167 https://doi.org/10.3115/1075096.1075117

[15] Koehn P, Och F J, Marcu D. Statistical phrase-based translation// Proceedings of the 2003 Conference of the North American Chapter of the Association for Computational Linguistics on Human Language Technology(HLT/NAACL). Edmonton,Canada,2003:48-54 https://doi.org/10.21236/ADA461156

\section{$7 \quad$ Author}

Weiwei Feng is with the School of Foreign Studies, Xi'an University, Xi'an, Shaanxi, China.

Article submitted 07 February 2018. Final acceptance 23 February 2018. Final version published as submitted by the author. 